\title{
Predição de ganhos genéticos em melhoramento florestal(1)
}

\author{
Rinaldo Cesar de Paula(2), Ismael Eleotério Pires ${ }^{(3)}$, Rita de Cássia Gonçalves Borges ${ }^{(3)}$ e Cosme Damião Cruz $^{(4)}$
}

Resumo - Foram avaliados os ganhos totais preditos por diferentes critérios de seleção, em seis características de crescimento em famílias de meios-irmãos de Eucalyptus camaldulensis. A situação simulada consistiu na seleção de $25 \%$ das 44 famílias estudadas, e de $17 \%$ das plantas dentro dessas famílias, proporcionando, assim, uma seleção de $4,25 \%$ dos indivíduos constantes do ensaio. Os critérios de seleção empregados foram: seleção direta e indireta; índice clássico de Smith \& Hazel, com quatro pesos econômicos, e índice de Pesek \& Baker, em três situações. Os critérios de seleção utilizados mostraram-se semelhantes entre si, com ligeira superioridade do índice clássico quando se estabeleceu como vetor de pesos econômicos o coeficiente de variação genética associado a cada uma das características analisadas.

Termos para indexação: Eucalyptus camaldulensis, seleção, índice de seleção, variação genética.

\section{Prediction of genetic gains in forest improvement}

\begin{abstract}
Total gains predicted by various selection criteria were evaluated, based on six characteristics of growth in half-sib families of Eucalyptus camaldulensis. The simulated situation consisted of selection of $25 \%$ among 44 families studied and of $17 \%$ of the plants in these families, totalizing a selection of $4.25 \%$ of the constant individuals of the essay. The selection criteria used were: direct and indirect selection; classical index of Smith \& Hazel, with four economic weights, and index of Pesek \& Baker, in three situations. The criteria used were similar to each other, with a slight superiority for the classical index when the genetic variation coefficient associated with each analysed characteristic was established as the vector of economic weights.
\end{abstract}

Index terms: Eucalyptus camaldulensis, selection, selection index, genetic variation.

\section{Introdução}

A possibilidade de prever ganhos é considerada uma das maiores contribuições da genética quantitativa para o melhoramento. Quando diferentes critérios de seleção são considerados, a predição de ganhos referentes a cada critério tem grande importância, pois orienta os melhoristas sobre como utilizar o material genético disponível da melhor maneira possível, visando à obtenção de ganhos máximos para as características de interesse.

(1) Aceito para publicação em 10 de agosto de 2001 .

Extraído da Tese de Doutorado, apresentada pelo primeiro autor à Universidade Federal de Viçosa (UFV), Viçosa, MG

(2) Universidade Estadual Paulista, Faculdade de Ciências Agrárias e Veterinárias, Dep. de Produção Vegetal, Via de Acesso Prof. Paulo Donato Castellene, s/n, CEP 14884-900, Jaboticabal, SP. E-mail: rcpaula@fcavunesp.br

(3) UFV, Dep. de Engenharia Florestal, CEP 36571-000 Viçosa, MG. E-mail: iepires@mail.ufv.br, elborges@mail.ufv.br

(4) UFV, Dep. de Biologia Geral, CEP 36571-000 Viçosa, MG E-mail: cdcruz@mail.ufv.br
A maneira mais fácil e prática de obter ganhos em relação a uma única característica é praticar a seleção diretamente sobre esta. Contudo, ao praticar a seleção visando a determinada característica, poderão ocorrer modificações em outras, cujo sentido e magnitude dependerão das características consideradas, e da associação entre estas. Outro fato, não menos importante, é que o melhoramento nem sempre visa à melhoria somente de uma característica, mas de múltiplas; e assim, deverão ser empregados outros critérios de seleção, para obter ganhos nas várias características de interesse, no sentido desejado.

A necessidade de realizar seleção com vistas a um conjunto de características de interesse para obter ganhos no sentido favorável a todas elas, simultaneamente, fez com que critérios de seleção, que não fossem baseados em apenas uma característica ou na seleção indireta, fossem implementados. Neste sentido, Baker (1986) e Cruz (1990) comentam 
que o índice de seleção constitui um caráter adicional, estabelecido pela combinação ótima de vários caracteres, e que se tem mostrado eficiente quando há necessidade de considerar simultaneamente várias características de interesse para conseguir as respostas desejadas. O índice de seleção é, pois, uma combinação de todas as características em apenas um índice (número) de cada unidade seletiva, praticando-se a seleção sobre os valores do índice e avaliando as respostas indiretas esperadas nas características originais. Embora investigações teóricas demonstrem a superioridade do índice de seleção em relação a outros critérios de seleção no intuito de obter múltiplas características (Vieira, 1988), o seu uso no setor florestal é ainda incipiente.

Os objetivos deste trabalho foram determinar a variabilidade genética entre e dentro de famílias de meios-irmãos de Eucalyptus camaldulensis Dehnh., em relação a características de crescimento, e predizer os progressos genéticos advindos da seleção direta e indireta e da adoção de índices de seleção.

\section{Material e Métodos}

Foram utilizados dados de um teste de progênie de E. camaldulensis Dehnh., com 67 meses de idade, instalado no Município de Paraopeba, MG. O ensaio, envolvendo 44 famílias de meios-irmãos, foi disposto no delineamento de blocos casualizados, com quatro repetições, espaçamento de $3,0 \times 1,5 \mathrm{~m}$, parcelas lineares de seis plantas, e bordadura geral simples.

Avaliaram-se as seguintes características: diâmetro à altura do peito (DAP, em $\mathrm{cm}$ ); altura comercial (ALTC, em m) até um diâmetro mínimo de $4 \mathrm{~cm}$; volume comercial com casca (VCCC, em m³ árvore); volume comercial sem casca (VCSC, em $\mathrm{m}^{3} /$ árvore); densidade básica da madeira (DENS, em $\mathrm{g} / \mathrm{cm}^{3}$ ); e biomassa do lenho (BIOML, em $\mathrm{kg}$ /árvore).

A ALTC e o DAP foram determinados por meio de medições feitas diretamente nas árvores. O VCCC e o VCSC foram determinados após cubagem rigorosa de todas as árvores, segundo as recomendações de Campos (1975). A DENS foi determinada, em laboratório, pelo processo do volume saturado, de acordo com Vital (1984), tomando-se um disco de madeira de $5 \mathrm{~cm}$ de espessura, ao nível do DAP, conforme recomendações de Finke Herrera (1989). A BIOML foi obtida pelo produto entre a DENS e o VCSC.
A análise estatística de cada característica, em plantas individuais foi realizada segundo Steel \& Torrie (1985), em que todos os efeitos, com exceção da média, foram considerados como aleatórios. Todos os procedimentos estatísticos foram processados de acordo com o programa computacional GENES (Cruz, 1997), como descrito em Paula (1997).

A seleção foi simulada, entre e dentro de famílias, conforme descrito em Cruz (1997), adotando-se três critérios de seleção: seleção direta sobre cada uma das características avaliadas; seleção com base no índice clássico (Smith, 1936; Hazel,1943) e seleção mediante o índice dos ganhos desejados (Pesek \& Baker, 1969).

$\mathrm{Na}$ seleção direta, avaliou-se também a conseqüente mudança nos demais caracteres não selecionados diretamente, pelo emprego de expressões apresentadas por Cruz \& Regazzi (1994).

Na seleção pelo índice clássico, ou índice de Smith \& Hazel (ISH), os vetores de pesos econômicos foram estabelecidos a partir dos próprios dados experimentais, conforme sugerido por Cruz (1990), e constaram de: $\mathrm{ISH}_{1}$ - estimativa do coeficiente de variação genética entre famílias $\left(\mathrm{CV}_{\mathrm{ge}}\right)$, relativo a cada uma das características avaliadas; $\mathrm{ISH}_{2}$ - estimativa do coeficiente de herdabilidade da média de famílias $\left(\mathrm{h}^{2} \mathrm{~m}\right)$, relativa às características em estudo; $\mathrm{ISH}_{3^{-}}$ estimativa do desvio-padrão genético $\left(\hat{\sigma}_{\text {ge }}\right)$, associado a cada característica; $\mathrm{ISH}_{4}$ - estimativa da correlação genética entre a BIOML e as demais características.

Os índices, com base nos ganhos desejados, ou índice de Pesek \& Baker (IPB), foram obtidos considerando-se o desvio-padrão genético entre famílias $\left(\hat{\boldsymbol{\sigma}}_{\mathrm{ge}}\right)$, relativo a cada característica, como vetor de ganhos desejados, estabelecido da seguinte maneira: $\mathrm{IPB}_{1}$ - foram consideradas todas as características como principais; $\mathrm{IPB}_{2}$ - apenas a BIOML foi considerada como principal; $\mathrm{IPB}_{3}$ - BIOML e DENS foram consideradas como características principais.

Na predição dos ganhos genéticos, em função dos critérios de seleção utilizados, foram adotadas como porcentagens de seleção $25 \%$ entre famílias e $17 \%$ dentro de famílias, com recombinação apenas das unidades selecionadas. A seleção foi simulada em relação a todas as características, no sentido de acréscimo em suas médias originais.

\section{Resultados e Discussão}

\section{Características gerais da população}

Todas as características avaliadas revelaram a existência de variações significativas entre famílias, a $1 \%$ de probabilidade, pelo teste F (Tabela 1 ). Esses resultados indicam a existência de variabilidade genética entre as famílias estudadas quanto a 
essas características, e sugerem a possibilidade de ganhos genéticos pela seleção das melhores famílias, corroborando os resultados obtidos por Kalil Filho et al. (1983), Santos et al. (1990), Silva (1990) e Cornacchia (1994), em seus estudos sobre outras espécies florestais. Isto evidencia que os materiais genéticos utilizados no setor florestal apresentam, de modo geral, grande variabilidade genética, e que as possibilidades de melhoramento desses materiais são promissoras. Esta observação concorda com as argumentações de Pires et al. (1996), de que a maioria dos materiais genéticos manipulados no setor florestal encontra-se em estádio selvagem, tornando possível a obtenção de ganhos expressivos, com pequeno esforço de seleção.

A DENS foi a característica que apresentou as maiores estimativas de herdabilidade, tanto entre famílias quanto entre indivíduos dentro de famílias (Tabela 1); estes resultados são concordantes com outros obtidos em diferentes espécies e condições de ambiente (Kageyama et al., 1983; Jourdain \& Olson, 1984; Almeida, 1993; Pires et al., 1996). Quanto às demais características analisadas, observaram-se valores expressivos e semelhantes entre si, no que diz respeito às estimativas de herdabilidade na média das famílias, o que evidencia um bom controle genético sobre elas. Tomados em conjunto, as estimativas de $h^{2}$ permitem predizer condições favoráveis para seleção de famílias, relativamente a todas as características em estudo. As estimativas de $\mathrm{h}_{\mathrm{d}}{ }_{\mathrm{d}}$ apresentaram a mesma tendência verificada $\mathrm{em} \mathrm{h}^{2}{ }_{\mathrm{m}}$, com maior valor de DENS, e estimativas semelhantes com relação às demais características.

As estimativas de correlação genotípica e fenotípica (Tabela 2) foram, em geral, de grandes magnitudes. As menores correlações foram as que envolveram a característica DENS, o que sugere que uma seleção nesta característica promoverá pequenas variações nas demais, e vice-versa. $\mathrm{O}$ fato de não existirem, no presente caso, correlações genotípicas desfavoráveis torna o processo seletivo mais simples, visto que modificações em uma característica tendem a ser acompanhadas de modificações, no mesmo sentido, em outras, e não necessitam, portanto, da adoção de restrições na seleção para obtenção de ganhos no sentido desejado. Houve tendência de os coeficientes de correlação genotípica superarem os de correlação fenotípica. Isto evidencia que os fatores genéticos são mais importantes que os de ambiente, na expressão de, pelo menos, um desses caracteres. Notam-se, também, valores expressivos para a correlação genotípica entre algumas características, o que leva à suspeita de ocorrência de genes pleiotrópicos atuando em seus controles.

As características avaliadas apresentaram um mesmo padrão de comportamento em face das variações ambientais. Isto é desejável, do ponto de vista

Tabela 1. Resumo da análise de variância e estimativas de alguns parâmetros genéticos referentes às características diâmetro à altura do peito (DAP), altura comercial (ALTC), volume comercial com casca (VCCC), volume comercial sem casca (VCSC), densidade básica da madeira (DENS) e biomassa do lenho (BIOML), em famílias de meios-irmãos de Eucalyptus camaldulensis, aos 67 meses de idade

\begin{tabular}{|c|c|c|c|c|c|c|c|}
\hline \multirow{2}{*}{$\begin{array}{l}\text { Fonte de } \\
\text { variação }^{(1)}\end{array}$} & \multirow[t]{2}{*}{ GL } & \multicolumn{6}{|c|}{ Quadrado médio } \\
\hline & & $\mathrm{DAP}(\mathrm{cm})$ & ALTC $(\mathrm{m})$ & VCCC ( $\mathrm{m}^{3}$ /árvore) & VCSC ( $\mathrm{m}^{3} /$ árvore $)$ & DENS $\left(\mathrm{g} / \mathrm{cm}^{3}\right)$ & BIOML (kg/árvore) \\
\hline Blocos & 3 & 31,779236 & 93,984742 & 0,010634 & 0,006789 & 0,001006 & $2.445,718925$ \\
\hline Famílias & 43 & $13,558640^{* * *}$ & $19,509427 * *$ & $0,003295 * *$ & $0,002075^{* *}$ & $0,006332 * *$ & $856,569634 * *$ \\
\hline Entre parcelas & 129 & 5,164839 & 7,689288 & 0,001139 & 0,000698 & 0,001287 & 270,363327 \\
\hline Dentro de parcelas & 880 & 4,497009 & 5,921238 & 0,001001 & 0,000621 & 0,001279 & 245,803736 \\
\hline Média & & 10,2838 & 11,9894 & 0,0691 & 0,0526 & 0,6008 & 31,8540 \\
\hline $\mathrm{CV}_{\exp }(\%)$ & & 9,021 & 9,442 & 19,933 & 20,526 & 2,437 & 21,074 \\
\hline $\mathrm{h}_{\mathrm{m}}^{2}$ & & 0,6191 & 0,6059 & 0,6543 & 0,6633 & 0,7967 & 0,6844 \\
\hline $\mathrm{h}_{\mathrm{d}}^{2}$ & & 0,2333 & 0,2495 & 0,2691 & 0,2770 & 0,4929 & 0,2981 \\
\hline $\mathrm{CV}_{\mathrm{ge}}$ & & 5,75 & 5,85 & 13,71 & 14,40 & 2,41 & 15,51 \\
\hline $\mathrm{CV}_{\mathrm{e}}$ & & 3,24 & 4,52 & 6,92 & 6,84 & 0,18 & 6,35 \\
\hline$\hat{\sigma}_{\mathrm{ge}}$ & & 0,5914 & 0,7018 & 0,0095 & 0,0076 & 0,0145 & 4,9422 \\
\hline
\end{tabular}


do melhoramento, pois a ação diferencial do ambiente sobre as variáveis envolvidas pode prejudicar o processo seletivo com base nos coeficientes de correlação (Castoldi, 1997).

A julgar pelos coeficientes de correlação obtidos, e tendo em vista que o interesse é aumentar os valores médios de cada característica analisada, pode-se considerar que há, de modo geral, perspectivas favoráveis para obtenção de ganhos via seleção indireta, para as características avaliadas, nestas condições.

\section{Expectativas de ganhos com a seleção}

Os maiores progressos obtidos mediante a seleção direta foram preditos em relação às seguintes características: BIOML, VCSC, VCCC, DAP, ALTC e DENS (Tabela 3). Se, por um lado, a DENS é a característica de maior herdabilidade, por outro, ela apresenta a menor expectativa de ganho com a seleção. Este fato é decorrente da baixa variabilidade genética apresentada por esse caráter, conforme pode ser evidenciado pelo baixo $\mathrm{CV}_{\mathrm{ge}}$ (Tabela 1). Quanto às

Tabela 2. Estimativas de correlações fenotípicas $\left(r_{f}\right)$, genotípicas $\left(\mathrm{r}_{\mathrm{g}}\right)$ e ambientais $\left(\mathrm{r}_{\mathrm{a}}\right)$, em média de famílias de meios-irmãos, referentes às características estudadas, em Eucalyptus camaldulensis, aos 67 meses de idade ${ }^{(1)}$

\begin{tabular}{|c|c|c|c|c|c|c|}
\hline Característica & Correlação & ALTC & VCCC & VCSC & DENS & $\overline{\text { BIOML }}$ \\
\hline \multirow[t]{3}{*}{ DAP } & $\mathrm{rf}$ & 0,8042 & 0,9524 & 0,9430 & 0,4388 & 0,9473 \\
\hline & $\mathrm{rg}$ & 0,8197 & 1,0026 & 0,9908 & 0,5262 & 0,9973 \\
\hline & $\mathrm{ra}$ & 0,7800 & 0,8663 & 0,8603 & 0,2486 & 0,8599 \\
\hline \multirow[t]{3}{*}{ ALTC } & $\mathrm{rf}$ & - & 0,8714 & 0,8829 & 0,3050 & 0,8593 \\
\hline & rg & - & 0,8594 & 0,8783 & 0,3526 & 0,8485 \\
\hline & ra & - & 0,8947 & 0,8951 & 0,2122 & 0,8872 \\
\hline \multirow[t]{3}{*}{ VCCC } & $\mathrm{rf}$ & - & - & 0,9950 & 0,3909 & 0,9905 \\
\hline & $\mathrm{rg}$ & - & - & 0,9946 & 0,4660 & 0,9921 \\
\hline & ra & - & - & 0,9960 & 0,2053 & 0,9889 \\
\hline \multirow[t]{3}{*}{ VCSC } & $\mathrm{rf}$ & - & - & - & 0,3663 & 0,9900 \\
\hline & $\mathrm{rg}$ & - & - & - & 0,4320 & 0,9895 \\
\hline & ra & - & - & - & 0,1997 & 0,9918 \\
\hline \multirow[t]{3}{*}{ DENS } & $\mathrm{rf}$ & - & - & - & - & 0,4819 \\
\hline & $\mathrm{rg}$ & - & - & - & - & 0,5454 \\
\hline & $\mathrm{ra}$ & - & - & - & - & 0,3126 \\
\hline
\end{tabular}

Pesq. agropec. bras., Brasília, v. 37, n. 2, p. 159-165, fev. 2002 demais características, as expectativas de ganhos com a seleção direta diferiram, principalmente em razão do $\mathrm{CV}_{\text {ge }}$ inerente a cada uma, uma vez que as estimativas de herdabilidade foram semelhantes entre si.

As respostas indiretas preditas em determinada característica, pela seleção direta praticada em outra, mostraram que a seleção indireta foi sempre inferior à seleção direta. Contudo, Falconer (1987) comentou que a seleção indireta poderá promover maiores progressos que a seleção direta, principalmente se o caráter auxiliar apresentar maior herdabilidade que o principal, e se a correlação genética entre ambos for de alta magnitude. Quando se utiliza, porém, a expressão de ganho genético a partir do diferencial de seleção, a seleção indireta será, no máximo, igual à direta, mas nunca superior.

Considerando o total dos ganhos promovido pela seleção direta em dada característica, e pelas respostas indiretas nas demais, a seleção praticada sobre o VCSC foi a que maximizou os ganhos genéticos, seguida, em ordem decrescente, por BIOML, VCCC, DAP, ALT e DENS. A eficiência da seleção indireta, via VCSC, foi de $95,00 \%$ para o DAP,

Tabela 3. Estimativas dos progressos genéticos com a seleção direta e indireta, índice clássico e índice com base nos ganhos desejados, entre e dentro de famílias de meios-irmãos, em Eucalyptus camaldulensis, aos 67 meses de $\operatorname{idade}^{(1)}$.

\begin{tabular}{lrrrrrrrr}
\hline Seleção & \multicolumn{7}{c}{ Resposta esperada } & $\begin{array}{c}\text { Total dos } \\
\text { ganhos }\end{array}$ \\
\cline { 2 - 7 } & DAP & ALTC & VCCC & VCSC & DENS & BIOML & \\
\hline DAP & 15,02 & 8,91 & 35,70 & 38,16 & 3,08 & 40,64 & 141,51 \\
ALTC & 9,08 & 12,12 & 29,89 & 32,58 & 0,59 & 32,82 & 117,08 \\
VCCC & 14,12 & 11,01 & 40,55 & 43,52 & 3,23 & 46,21 & 158,64 \\
VCSC & 14,27 & 10,87 & 40,49 & 43,65 & 3,34 & 46,48 & 159,10 \\
DENS & 7,11 & 4,28 & 18,00 & 18,13 & 7,48 & 23,85 & 78,85 \\
BIOML & 14,16 & 10,75 & 40,28 & 43,37 & 3,66 & 46,50 & 158,71 \\
ISH $_{1}$ & 14,40 & 10,53 & 40,61 & 43,79 & 3,98 & 47,18 & 160,49 \\
ISH $_{2}$ & 13,78 & 10,57 & 39,43 & 42,68 & 3,94 & 46,01 & 156,41 \\
ISH $_{3}$ & 13,82 & 10,50 & 39,37 & 42,49 & 4,10 & 45,96 & 156,24 \\
ISH $_{4}$ & 13,78 & 10,57 & 39,43 & 42,68 & 3,94 & 46,01 & 156,41 \\
IPB $_{1}$ & 8,85 & 4,17 & 15,12 & 15,71 & 2,48 & 16,39 & 62,72 \\
IPB $_{2}$ & 13,82 & 10,50 & 39,37 & 42,49 & 4,10 & 45,96 & 156,24 \\
IPB $_{3}$ & 14,16 & 10,75 & 40,28 & 43,37 & 3,66 & 46,50 & 158,71 \\
\hline
\end{tabular}

${ }^{(1)} \mathrm{ISH}_{(1.4)}$ : índice clássico (Smith \& Hazel); $\mathrm{IPB}_{(1.3)}$ : índice com base nos ganhos desejados (Pesek \& Baker); DAP: diâmetro à altura do peito; ALTC: altura comercial; VCCC: volume comercial com casca; VCSC: volume comercial sem casca; DENS: densidade básica da madeira; BIOML: biomassa do lenho. 
89,63\% para ALTC, 99,85\% para VCCC, $44,65 \%$ para DENS e $99,96 \%$ para BIOML, em relação à seleção direta praticada sobre cada uma destas características. Tais resultados evidenciam boas perspectivas do melhoramento dessas características, nestas condições.

$\mathrm{O}$ índice clássico mostrou-se pouco flexível e apresenta pequenas alterações no total dos ganhos, mesmo com a variação nos pesos econômicos estabelecidos (Tabela 4), proporcionando, por conseguinte, estimativas semelhantes de ganhos nas características originais (Tabela 3 ). $\mathrm{O} \mathrm{ISH}_{1}$ possibilitou a previsão de maiores progressos genéticos do que os demais índices e a seleção direta, embora os ganhos, em cada caráter sob seleção direta, tenham sido superiores aos preditos por este índice. Cruz (1990) relatou que a seleção direta, apesar de proporcionar a maximização dos ganhos individuais, não possibilita a obtenção de ganhos em níveis satisfatórios nos demais caracteres. Assim, mesmo sendo menores os ganhos em cada caráter, o índice mostra-se mais eficiente que a seleção direta, por proporcionar maiores ganhos totais, distribuindo-os melhor entre os vários caracteres. Este índice $\left(\mathrm{ISH}_{1}\right)$ foi construído considerando-se como vetor de pesos econômicos o $\mathrm{CV}_{\text {ge }}$ de cada característica. Este se mostrou como valor adequado no estabelecimento de pesos econômicos na construção do índice clássico, nestas condições. Cruz \& Regazzi (1994) argumentaram que o $\mathrm{CV}_{\mathrm{ge}}$ apresenta propriedades interessantes, por ser adimensional e diretamente proporcional à variabilidade genética existente na população.

Considerando-se todas as características como principais ( $\mathrm{IPB}_{1}$ ), o ganho genético total predito mediante seleção pelo índice de Pesek \& Baker foi o menor entre os índices, sugerindo que no processo seletivo deve-se priorizar determinadas características em detrimento de outras. $\mathrm{O} \mathrm{IPB}_{3}$, que considerou tanto a BIOML quanto a DENS como princi-

Tabela 4. Pesos econômicos (PE), coeficientes de ponderação do índice entre $(\mathrm{CPa})$ e dentro $(\mathrm{CPb})$ de famílias e ganhos desejados (GD) estabelecidos, com base no índice clássico de Smith \& Hazel e no índice de ganhos desejados de Pesek \& Baker, referentes às características em estudo ${ }^{(1)}$.

\begin{tabular}{lccrrrrr}
\hline Índice & Estatística & DAP & ALTC & \multicolumn{1}{c}{ VCCC } & \multicolumn{1}{c}{ VCSC } & \multicolumn{1}{c}{ DENS } & BIOML \\
\hline $\mathrm{ISH}_{1}$ & $\mathrm{PE}$ & 5,7507 & 5,8534 & 13,7119 & 14,4041 & 2,4131 & 15,5151 \\
& $\mathrm{CPa}$ & 8,9230 & $-15,2155$ & $-6.675,2168$ & $10.897,4873$ & 803,7084 & 7,6392 \\
& $\mathrm{CPb}$ & 8,2157 & $-11,6594$ & $-4.432,9751$ & $11.283,7539$ & $1.390,8819$ & $-4,3964$ \\
$\mathrm{ISH}_{2}$ & $\mathrm{PE}$ & 0,6191 & 0,6059 & 0,6543 & 0,6633 & 0,7967 & 0,6844 \\
& $\mathrm{CPa}$ & 0,3633 & $-0,6078$ & $-316,1181$ & 587,2719 & 44,2969 & 0,2580 \\
& $\mathrm{CPb}$ & 0,3738 & $-0,4993$ & $-231,3539$ & 602,0761 & 70,1362 & $-0,2714$ \\
$\mathrm{ISH}_{3}$ & $\mathrm{PE}$ & 0,5914 & 0,7018 & 0,0095 & 0,0076 & 0,0145 & 4,9422 \\
& $\mathrm{CPa}$ & 2,9298 & $-5,0441$ & $-2.047,7609$ & $3.104,3840$ & 227,2774 & 2,6905 \\
& $\mathrm{CPb}$ & 2,5745 & $-3,7592$ & $-1.286,1058$ & $3.232,8411$ & 413,3445 & $-1,1382$ \\
& $\mathrm{PE}$ & 0,9973 & 0,8485 & 0,9921 & 0,9895 & 0,5454 & 1,0000 \\
$\mathrm{ISH}_{4}$ & $\mathrm{CPa}$ & 0,5306 & $-0,9245$ & $-458,4941$ & 858,9751 & 64,6683 & 0,3778 \\
& $\mathrm{CPb}$ & 0,5502 & $-0,7464$ & $-335,2296$ & 876,6251 & 102,6231 & $-0,3939$ \\
& $\mathrm{GD}$ & 0,5914 & 0,7018 & 0,0095 & 0,0076 & 0,0145 & 4,9422 \\
$\mathrm{IPB}_{1}$ & $\mathrm{CPa}$ & 8,1568 & $-0,0290$ & $-308,4145$ & $2.338,4885$ & 218,5310 & $-4,0763$ \\
& $\mathrm{CPb}$ & 1,5429 & $-0,3434$ & $-163,0428$ & $1.223,6805$ & 122,3720 & $-1,7596$ \\
& $\mathrm{GD}$ & 0,0000 & 0,0000 & 0,0000 & 0,0000 & 0,0000 & 4,9422 \\
$\mathrm{IPB}_{2}$ & $\mathrm{CPa}$ & 0,1636 & 12,2829 & 168,1017 & $-115,6841$ & $-0,3013$ & 0,1725 \\
& $\mathrm{CPb}$ & $-0,0574$ & 23,0069 & 175,2306 & $-70,0919$ & $-0,2205$ & 0,1481 \\
& $\mathrm{GD}$ & 0,0000 & 0,0000 & 0,0000 & 0,0000 & 0,0145 & 4,9422 \\
$\mathrm{IPB}_{3}$ & $\mathrm{CPa}$ & 55,4064 & $-0,0791$ & 197,2587 & $-35,0592$ & $-0,3403$ & 0,0115 \\
& $\mathrm{CPb}$ & 43,7344 & $-0,1252$ & 106,1102 & 14,8382 & $-0,3564$ & 0,1916 \\
\hline
\end{tabular}

${ }^{(1)} \mathrm{ISH}_{\mathrm{i}(\mathrm{i}=1, \ldots, 4)}$ : índice de Smith \& Hazel; $\mathrm{IPB}_{\mathrm{i}(\mathrm{i}=1,2,3)}$ : índice de Pesek \& Baker; DAP: diâmetro à altura do peito; ALTC: altura comercial; VCCC volume comercial com casca; VCSC: volume comercial sem casca; DENS: densidade básica da madeira; BIOML: biomassa do lenho. 
pais, foi o que proporcionou, entre os índices, com base nos ganhos desejados, a maior expectativa de ganho genético total, sendo superior ao $\mathrm{IPB}_{2}$, que considerou como principal apenas a BIOML.

$\mathrm{O}$ índice com base nos ganhos desejados mostrou-se mais flexível que o índice clássico, tendo identificado diferentes famílias e indivíduos à medida que a importância das características envolvidas fora alterada. Todavia, esse índice não se mostrou tão eficiente quanto o índice clássico, e predisse ganhos genéticos, em geral inferiores aos preditos pelo índice de Smith \& Hazel. O IPB 2 e o $\mathrm{ISH}_{3}$ proporcionaram a mesma expectativa de ganhos, e as perspectivas de ganhos com a utilização do $\mathrm{IPB}_{3}$ foram as mesmas que as estimadas mediante a seleção direta em BIOML.

Em famílias de irmãos-germanos de milho, o índice clássico tem sido superior ao índice de Pesek \& Baker (Cruz, 1990). Segundo este autor, o índice de Pesek \& Baker só proporcionou resultados satisfatórios quando foi estabelecido como ganho desejado um desvio-padrão genético para cada característica. Wells \& Kofoid (1986) e Castoldi (1997) constataram baixa eficiência do índice de Pesek \& Baker em trigo e em milho, respectivamente. $\mathrm{O}$ índice clássico mostrou-se adequado em várias situações simuladas (Castoldi, 1997); e entre os pesos econômicos estabelecidos, aquele que correspondeu às estimativas dos efeitos diretos obtidos em análise de trilha foi o mais promissor.

Sendo a BIOML e a DENS características de maior importância quando o objetivo é a produção de carvão, por serem elas diretamente relacionadas com o teor de carbono fixo (Vital et al., 1994), o índice de seleção clássico $\left(\mathrm{ISH}_{1}\right)$ é preferível em relação aos demais critérios adotados, por proporcionar maiores progressos nessas características.

\section{Conclusões}

1. A grande variabilidade genética, em geral inerente aos materiais genéticos utilizados na área florestal, possibilita boas perspectivas de melhoramento pela seleção direta e indireta.

2. O índice com base nos ganhos desejados (Pesek \& Baker) proporciona resultados semelhantes aos de outros critérios de seleção empregados.
3. O índice de seleção clássico (Smith \& Hazel) mostra-se promissor para o melhoramento de múltiplas características, por superar outros critérios de seleção, em termos de ganhos totais, quando se estabelece como peso econômico em cada característica o equivalente ao coeficiente de variação genética entre famílias.

\section{Referências}

ALMEIDA, J. Características de crescimento e qualidade da madeira de progênies de Eucalyptus camaldulensis Dehnh. Viçosa, MG: UFV, 1993. 63 p. Dissertação de Mestrado.

BAKER, R. J. Selection indices in breeding. Boca Raton: CRC, 1986. 218 p

CAMPOS, J. C. C. Dendrometria. Viçosa, MG: UFVImprensa Universitária, 1975. Pt. 1, 64 p.

CASTOLDI, F. L. Comparação de métodos multivariados aplicados na seleção em milho. Viçosa, MG: UFV, 1997. 118 p. Tese de Doutorado.

CORNACCHIA, G. Variabilidade genética em procedências de $P$. caribaea var. hondurensis Barr. \& Golf., P. oocarpa Schiede e P. tecunumanii (Schw.) Eguiluz \& Perry na região oeste do Estado da Bahia. Viçosa, MG: UFV, 1994. 155 p. Dissertação de Mestrado.

CRUZ, C. D. Aplicação de algumas técnicas multivariadas no melhoramento de plantas. Piracicaba: Esalq, 1990. 188 p. Tese de Doutorado.

CRUZ, C. D. Programa Genes: aplicativo computacional em genética e estatística. Viçosa, MG: Ed. da UFV, 1997. $442 \mathrm{p}$

CRUZ, C. D.; REGAZZI, A. J. Modelos biométricos aplicados ao melhoramento genético. Viçosa, MG: UFV-Imprensa Universitária, 1994. 390 p.

FALCONER, D. S. Introdução à genética quantitativa Trad. de Martinho de Almeida e Silva e José Carlos Silva. Viçosa, MG: UFV-Imprensa Universitária, 1987. 279 p.

FINKE HERRERA, M. E. Densidade básica e equações de peso de madeira seca de povoamentos de eucaliptos de acordo com a idade, local, espécie e método de regeneração. Viçosa, MG: UFV, 1989. 113 p. Dissertação de Mestrado.

HAZEL, L. N. The genetic basis for constructing selection indexes. Genetics, New York, v. 28, n. 6, p. 476-490, 1943. 
JOURDAIN, C. J.; OLSON, J. R. Wood property variation among forty-eight families ofAmerican sycamore Wood and Fiber Science, Madison, v. 16, n. 4, p. 498506,1984

KAGEYAMA, P. Y.; MORA, A. L.; BARRICHELO, L. E. G.; MIGLIORINI, A. J.; SANSIGOLO, C. A. Variação genética para densidade da madeira em progênies de Eucalyptus grandis. Silvicultura, São Paulo, v. 8, n. 28, p. 318-324, 1983.

KALIL FILHO, A. N.; PIRES, C. L. S.; FONTES, M. A. Análise do comportamento e estimação de parâmetros genéticos em progênies de Pinus elliottii Engelm. var. elliottii na região de Itararé (SP). Silvicultura, São Paulo, v. 8 , n. 28 , p. $325-326,1983$

PAULA, R. C. Avaliação de diferentes critérios de seleção aplicados em melhoramento florestal. Viçosa, MG: UFV, 1997. 74 p. Tese de Doutorado.

PESEK, J.; BAKER, R. J. Desired improvement in relation to selection indices. Canadian Journal of Plant Science, Ottawa, v. 49, p. 803-804, 1969

PIRES, I. E.; CRUZ, C. D.; BORGES, R. C. G.; REGAZZI,A. J. Índice de seleção combinada aplicado ao melhoramento genético de Eucalyptus spp. Revista Árvore, Viçosa, v. 20, n. 2, p. 191-197, 1996

SANTOS, P. E. T.; GARCIA, C. H.; MORI, E. S.; MORAES, M. L. T. Potencial para programas de melhoramento, estimativas de parâmetros genéticos e interação progênies x locais em populações de Eucalyptus urophylla S. T. Blake. IPEF, Piracicaba, n. 43/44, p. 1119, 1990

SILVA, J. F. Variabilidade genética em progênies de Eucalyptus camaldulensis Dehnh. e sua interação com espaçamentos. Viçosa, MG: UFV, 1990. 110 p. Dissertação de Mestrado.

SMITH, H. F. A discriminant function for plant selection Annals of Eugenics, Cambridge, Inglaterra, v. 7, p. 240 250, 1936

STEEL, R. G. D.; TORRIE, J. H. Bioestadística; principios y procedimientos. 2. ed. Bogotá: McGraw Hill, 1985. $622 \mathrm{p}$.

VIEIRA, J. V. Herdabilidades, correlações e índice de seleção em populações de cenoura (Daucus carota $\mathbf{L}$.) Viçosa, MG: UFV, 1988. 86 p. Tese de Doutorado.

VITAL, B. R. Métodos de determinação da densidade da madeira. Viçosa, MG: Sociedade de Investigações Florestais, 1984. 21 p. (Boletim Técnico, 1).

VITAL, B. R.; ALMEIDA, J.; VALENTE, O. F.; PIRES, I. E. Características de crescimento das árvores e da qualidade da madeira de Eucalyptus camalduensis para a produção de carvão. IPEF, Piracicaba, n. 47, p. 22-28, 1994.

WELLS, W. C.; KOFOID, K. D. Selection indices to improve an intermating population of spring wheat. Crop Science, Madison, v. 26, p. 1104-1109, 1986. 